\title{
Investigation of flow instability characteristics in a low specific speed centrifugal pump using a modified partially averaged Navier-Stokes model
}

Proc IMechE Part A:

$J$ Power and Energy

$0(0) \mathrm{I}-15$

(C) IMechE 2019

Article reuse guidelines:

sagepub.com/journals-permissions DOI: 10.1 I77/09576509|9830188 journals.sagepub.com/home/pia

@SAGE

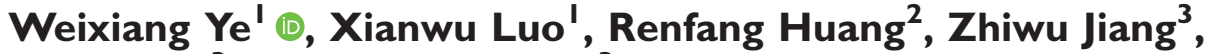 \\ Xiaojun $\mathrm{Li}^{3}$ (1) and Zuchao Zhu ${ }^{3}$
}

\begin{abstract}
In this study, a modified partially averaged Navier-Stokes (MPANS) model is applied to investigate the flow instability characteristics in a low specific centrifugal pump. In MPANS model, the unresolved-to-total ratio of kinetic energy $f_{k}$ is determined according to the local grid size and turbulence length scale. The numerical results by MPANS model are compared with that simulated by SST $k-\omega$ model and the available experimental data. It is noted that MPANS model shows better performance for investigating the unstable flow in the current pump under part-load operation conditions. The timeaveraged internal flow and flow incidence in the pump impeller depicts that with the decreasing flow coefficient, flow separation develops in the impeller. Owing to the strong separation flow as well as vortex evolution, incidence angle is large and varies remarkably at the entrance of blade-to-blade passage in the pump impeller. The evolution dynamics of rotating stall is further discussed in detail based on vorticity transport equation. During the evolution of rotating stall, the vortex stretching term has an important effect on vorticity transport under the part-load conditions. The analysis of the pressure fluctuations excited by periodic evolution of rotating stall shows that the rotating stall cell propagates along the rotational direction, and identifies the rotating stall frequency $\left(f_{\text {stall }}\right)$, which is much lower than the rotational frequency of the impeller, $f_{\mathrm{n}}\left(f_{\text {stall }}=8.76 \% f_{\mathrm{n}}\right)$. Finally, two-dimensional Lagrangian coherent structure (LCS) is used to reveal the separation flow in blade-to-blade passages of the pump by monitoring the trajectory of the particles. Both LCS and vortex structure by $\lambda_{2}$ can clearly demonstrates the passage blockage and flow separation under the part-load operation conditions, depicting that the separation flow occurs at blade suction side and develops from the leading edge to the main passage in the impeller.
\end{abstract}

\section{Keywords \\ Centrifugal pump, MPANS, flow separation, rotating stall, Lagrangian coherent structures}

Date received: 10 October 2018; accepted: 21 January 2019

\section{Introduction}

Much attention has been paid to the flow instability such as rotating stall, rotating cavitation, surge, etc. in fluid machinery for decades. ${ }^{1}$ As one of the flow instability phenomena, positive slope is quite common when pumps or pump-turbines at pump mode are operated under off-design conditions. It is acknowledged that the formation of positive slope is caused by rotating stall, which induces flow separation, reverse flow and flow blockage in vane passages. Usually under the operation condition with positive slope, there will be strong pressure fluctuations in pumps. Up to now, many researches have been conducted to investigate such flow instability extensively. Comprehensive reviews on this topic have been given. ${ }^{2,3}$
In the past years, numerous experiments had been conducted to investigate the rotating stall and pressure fluctuations especially in a pump or a pump-turbine. Ran et al. ${ }^{4}$ studied the pressure

\footnotetext{
'State Key Laboratory of Hydroscience and Engineering, Department of Energy and Power Engineering, Tsinghua University, Beijing, China ${ }^{2}$ Key Laboratory for Mechanics in Fluid Solid Coupling System, Institute of Mechanics, Chinese Academy of Sciences, Beijing, China ${ }^{3}$ Key Laboratory of Fluid Transmission Technology of Zhejiang Province, Zhejiang Sci-Tech University, Hangzhou, China
}

\section{Corresponding author:}

Xianwu Luo, State Key Laboratory of Hydroscience and Engineering, Department of Energy and Power Engineering, Tsinghua University, Beijing 100084, China.

Email: luoxw@tsinghua.edu.cn 
fluctuations in a pump turbine under large partial flow conditions by model test and found that there were two separated positive slopes at the characteristic curve of the pump turbine at pump mode. By measuring the pressure fluctuations located at guide vanes, the authors found the pressure fluctuations at the unstable operating points had a dominant component with the lower frequency than the shaft rotation, i.e., 0.2 times of shaft rotating frequency, which was induced by the rotating stall in the flow passage. Ullum et al. $^{5}$ analyzed the rotating stall based on experimental data. The results indicate that nearly identical frequencies of rotating stall can be observed in both velocity and pressure power spectra. Krause et al. ${ }^{6}$ investigated different stages of the developing rotating stall in a radial pump by using particle-image velocimetry (PIV), and a spatially stable rotating stall cell was observed under certain flow discharge, which started to rotate inside the impeller if the flow rate decreased further.

With the development of the computation technology and computational fluid dynamic, it becomes possible to study flow instability by numerical simulation. For the simulations of flow instability, the turbulence modeling method is crucial because the flow in positive slope regions is inevitably unsteady, and the complicated flow structure is hard to capture accurately. Reynolds average Navier-Stokes (RANS), ${ }^{7,8}$ model is popular, which has been widely utilized in many engineering applications. Since RANS method always over-estimates the viscous effect, some modifications are necessary to improve the prediction accuracy. ${ }^{9}$ On the other hand, large eddy simulation $(\mathrm{LES})^{10,11}$ can capture unsteady details during rotating stall occurrence, and is preferable for some engineering applications. Nevertheless, the cost of computing resource and the requirement for mesh quality are not affordable in most engineering applications. As a bridge from RANS to Direct numerical simulation (DNS), partially averaged Navier-Stokes (PANS) has been introduced and applied. ${ }^{12-14}$ For better accuracy, PANS model has been modified by controlling two parameters, i.e., the unresolvedto-total ratios of kinetic energy $f_{\mathrm{k}}$ and dissipation $f_{\varepsilon}$. Girimaji and Abdol-Hamid proposed a two-stage PANS model with $f_{\mathrm{k}}$ varying in space throughout an unsteady flow simulation. ${ }^{15}$ In order to make turbulence model suitable for the complicated applications, a modified partially averaged Navier-Stokes model (MPANS) is proposed to solve the industrial flows in turbo-machines that often encounter with flow separation, recirculation and reattachment. ${ }^{16}$

Although a lot of researches have been focused on the structure of rotating stall cells and the evolution, most of them are based on the Eulerian perspective. In recent years, some applications of Lagrangian coherent structure (LCS) show interesting performance to depict the flow structure. LCS was first utilized by Haller and Yuan, ${ }^{17}$ who defined LCS as ridges of the Finite-Time Lyapunov Exponent (FTLE) field to visualize the complicated flow. These ridges are adopted to describe the fluid dynamics phenomenon in a fully developed turbulence flow. Franco et al. ${ }^{18}$ analyzed the structure of flow field around a cylinder under low Reynolds number. The results showed that LCS had a better performance in capturing the unsteady vortex region compared to the Eulerian system. Green et al. ${ }^{19}$ calculated the 3D turbulent flow using LCS method, which indicates that LCS is possible to define the flow details and vortex structures. In addition, LCS has been used in many engineering cases, such as the flows around a circular cylinder ${ }^{20}$ and 2D hydrofoil. ${ }^{21,22}$ Long et al. ${ }^{23}$ adopted three-dimensional LCS to reveal the influence of U-type structures on local flow, and their results showed that there was a close relationship between flow separation and vortex structures.

Inspired by the previous work, the present paper aims to analyze the instability characteristics of a centrifugal pump with low specific speed to investigate the mechanism of the positive slope phenomenon by using MPANS model. For comparisons, the simulation by SST $k-\omega$ turbulence model and PIV test are also performed. Further, two-dimensional LCS is introduced to analyze the internal flow so as to reveal the mechanism of flow separation during positive slope phenomenon. This paper is organized as follows. The governing equations and partially averaged NavierStokes approach are introduced first. Problem setup, mesh and boundary conditions are described next. Then, hydraulic performance and internal flow simulated by a modified PANS model are illustrated, followed by various aspects of the unsteady flow predicted by the modified PANS model.

\section{Governing equations and MPANS model}

The mass and momentum conservation equations for the unsteady flow can be expressed as

$$
\begin{aligned}
& \frac{\partial \rho}{\partial t}+\frac{\partial\left(\rho u_{j}\right)}{\partial x_{j}}=0 \\
& \frac{\partial\left(\rho u_{i}\right)}{\partial t}+\frac{\partial}{\partial x_{j}}\left(\rho u_{i} u_{j}\right)=-\frac{\partial p}{\partial x_{i}}+\frac{\partial}{\partial x_{j}}\left[\left(\mu+\mu_{t}\right)\left(\frac{\partial u_{i}}{\partial x_{j}}+\frac{\partial u_{j}}{\partial x_{i}}\right)\right]
\end{aligned}
$$

where $\rho$ is the density, $p$ is the pressure. $u_{\mathrm{i}}$ is the velocity in the $i$-th direction, while $u_{\mathrm{j}}$ is the velocity in the $j$-th direction. $\mu$ and $\mu_{\mathrm{t}}$ represent the laminar and turbulence dynamic viscosity.

In MPANS model with standard $k-\varepsilon$ model as the parent model, the eddy kinematic viscosity $v_{\mathrm{u}}$ is formulated by $v_{\mathrm{u}}=C_{\mu} k_{\mathrm{u}}^{2} / \varepsilon_{\mathrm{u}}$, where the unresolved turbulent kinetic energy $k_{\mathrm{u}}$ and turbulent dissipation rate $\varepsilon_{\mathrm{u}}$ are determined by two parameters, i.e., the unresolved-to-total ratios of kinetic energy $f_{k}$ and 
dissipation $f_{\varepsilon}$ respectively. The two parameters are defined in equation (3).

$$
f_{k}=\frac{k_{u}}{k}, \quad f_{\varepsilon}=\frac{\varepsilon_{u}}{\varepsilon}
$$

The governing equations for kinetic energy and dissipation are described in equations (4) and (5).

$$
\begin{aligned}
& \frac{\partial k_{u}}{\partial t}+U_{j} \frac{\partial k_{u}}{\partial x_{j}}=P_{u}-\varepsilon_{u}+\frac{\partial}{\partial x_{j}}\left[\left(\nu+\frac{v_{u}}{\sigma_{k u}}\right) \frac{\partial k_{u}}{\partial x_{j}}\right] \\
& \frac{\partial \varepsilon_{u}}{\partial t}+U_{j} \frac{\partial \varepsilon_{u}}{\partial x_{j}}=C_{e 1} \frac{P_{u} \varepsilon_{u}}{k_{u}}-C_{e 2}^{*} \frac{\varepsilon_{u}^{2}}{k_{u}}+\frac{\partial}{\partial x_{j}}\left[\left(\nu+\frac{v_{u}}{\sigma_{\varepsilon u}}\right) \frac{\partial \varepsilon_{u}}{\partial x_{j}}\right]
\end{aligned}
$$

where $P_{\mathrm{u}}$ is unresolved production term. The model constants are $C_{\varepsilon 1}=1.44, \quad C_{\varepsilon 2}=1.92, \quad \sigma_{\mathrm{k}}=1.0$ and $\sigma_{\varepsilon}=1.3$. Other coefficients are

$$
\begin{aligned}
\sigma_{k u} & =\sigma_{k} \frac{f_{k}^{2}}{f_{\varepsilon}} ; \quad \sigma_{\varepsilon u}=\sigma_{\varepsilon} \frac{f_{k}^{2}}{f_{\varepsilon}} ; \\
C_{e 2}^{*} & =C_{e 1}+\frac{f_{k}}{f_{\varepsilon}}\left(C_{e 2}-C_{e 1}\right)
\end{aligned}
$$

For the flows with high Reynolds number, $f_{\varepsilon}$ is generally set to 1.0 , since there is a clear separation between the energy containing and dissipation ranges. ${ }^{16}$ The unresolved-to-total kinetic energy $f_{k}$, can be expressed as equation (6).

Table I. Specifications of the test pump.

\begin{tabular}{ll}
\hline Parameter & Value \\
\hline Impeller inlet diameter $D_{1}$ & $56 \mathrm{~mm}$ \\
Impeller exit diameter $D_{2}$ & $142 \mathrm{~mm}$ \\
Blade width at impeller exit $b_{2}$ & $7 \mathrm{~mm}$ \\
Blade number $N$ & 5 \\
Shaft rotational speed $n$ & $1000 \mathrm{r} / \mathrm{min}$ \\
Flow rate at design point $Q_{\mathrm{d}}$ & $1.8 \mathrm{~m}^{3} / \mathrm{h}$ \\
Pump head at design point $H_{\mathrm{d}}$ & $2.67 \mathrm{~m}$ \\
\hline
\end{tabular}

$$
f_{k}=\min \left(1, \frac{1}{\sqrt{C_{\mu}}}\left(\frac{\Delta}{\Lambda}\right)^{\frac{2}{3}}\right)
$$

where $C_{\mu}$ is model coefficient. $\Lambda$ is the Taylor turbulence length scale, which is defined as $\Lambda=k^{1.5} / \varepsilon . \Delta$ stands for the local grid size, defined as $\Delta=\left(\Delta x^{*}\right.$ $\left.\Delta y^{*} \Delta z\right)^{1 / 3}$.

In the present analysis, the equations are solved by using the commercial CFD code CFX 15.0.

\section{Problem description}

\section{Computation domain}

The test pump with specific speed of $39.12 \mathrm{~m} \cdot \mathrm{min}^{-1} \cdot \mathrm{m}^{3} \mathrm{~s}^{-1}$ has a shrouded centrifugal impeller. Some specifications of the pump are list in Table 1. Figure 1 shows the computation domain including inlet pipe, impeller, casing, outlet pipe, pipe extension, etc. Because there is a clearance with $2 \mathrm{~mm}$ width between pump cover and impeller hub/ shroud, a zone is formed and named as "gap", which is rotating domain shown in Figure 1.

\section{Mesh generation and independence test}

For each flow component shown at Figure 1, structured meshes are generated.

Five mesh resolutions were tested by monitoring head coefficient $(\Psi)$ and hydraulic efficiency $(\eta)$ of the pump, which are defined as equations (7) and (8), respectively.

$$
\begin{gathered}
\psi=\frac{H}{u_{2}^{2}(2 g)} \\
\eta=\frac{\rho g Q H}{P_{\text {in }}}
\end{gathered}
$$

where $H$ is pump head, and $Q$ represents flow rate. $u_{2}$ is the peripheral speed at impeller exit. $P_{\text {in }}$ represents the power input to the pump shaft.

For PANS method, lower value of $f_{\mathrm{k}}$ could reach more accurate results. Once the mesh yields a meshindependent solution for the case of $f_{\mathrm{k}}=0.2$, which is

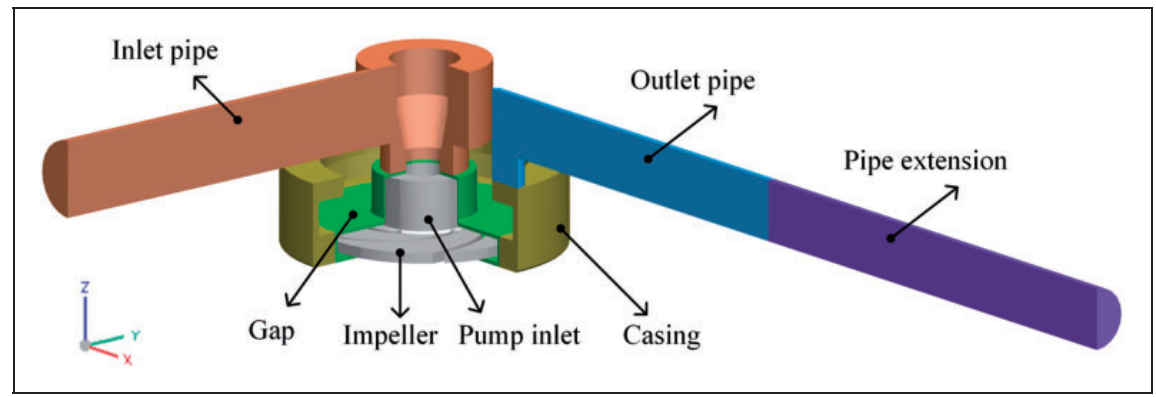

Figure I. Computation domain. 
the smallest $f_{\mathrm{k}}$ value in this study, it can support any simulation with higher $f_{\mathrm{k}}\left(f_{\mathrm{k}}>0.2\right)$ resolution. Thus, five sets of mesh schemes are performed for mesh independence test using $f_{\mathrm{k}}=0.2$. The relations of head coefficient and hydraulic efficiency versus mesh number are shown in Figure 2. Note that the mesh of totally 3.34 million grid points results in the similar predictions as the mesh of 3.7 million grid points. It should be noted that any further grid refinement leads to relatively more complex vortex structures, which will require more computational resources. Thus, the mesh of totally 3.34 million grid points was employed as the final mesh scheme in the following simulations.

The mesh scheme with 3.34 million grid points is shown in Figure 3. The refined mesh near the impeller blade is carefully treated to satisfy the requirement of $\mathrm{y}^{+}$value. The values of $\mathrm{y}^{+}$at the wall surface for impeller blade, hub and shroud are listed in Table 2.

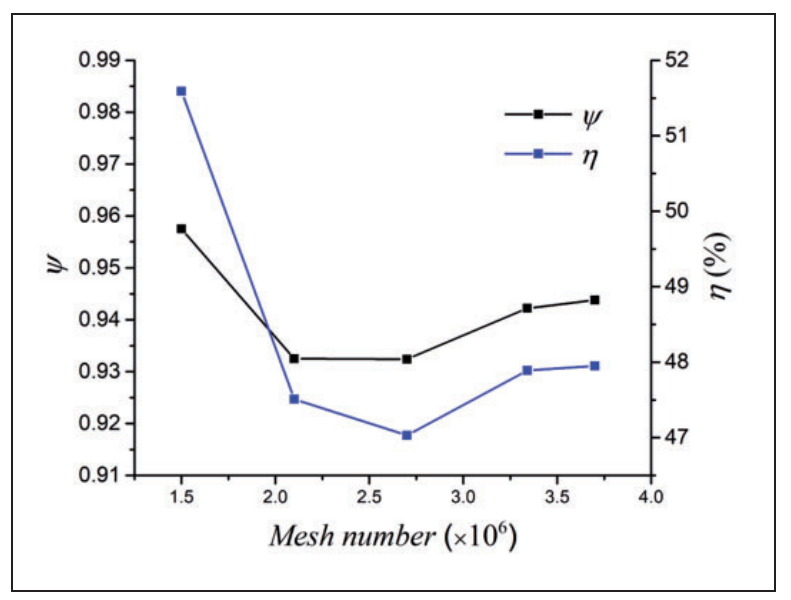

Figure 2. Mesh independence test $\left(f_{k}=0.2\right)$.
It is noted that the largest value of $\mathrm{y}^{+}$is below 200, which meets the requirements of MPANS model.

Figure 4 displays $f_{k}$-field with the mesh resolution of 3.34 million grid points. It is clear that the value of $f_{k}$ is larger near the wall surface, and that is smaller in the main stream of blade-to-blade passage. The minimum $f_{k}$ is 0.2 . For better accuracy, the simulation results with $f_{k}=0.2$ has been used for mesh independence test.

\section{Simulation setup}

The time-dependent governing equations were discretized spatially and temporally. The high order resolution scheme was used for the advection term, and the second-order backward Euler method is used for transient simulation. Water at $25^{\circ} \mathrm{C}$ is selected as the fluid. A rotational coordinate system is set for the area of pump impeller and the gap, and a stationary system is set for the stationary components. Interfaces between those connecting faces are introduced, where the sliding mesh strategy is applied. For boundary conditions, a mass flow rate is designated at the inlet of computation domain, and total pressure is set at the domain outlet. No slip boundary conditions are imposed on all solid walls. The simulations have been conducted based on MPANS turbulence model with $k-\varepsilon$ model as the parent model. The time step is

Table 2. $\mathrm{y}^{+}$Distribution near wall surfaces.

\begin{tabular}{llll}
\hline Component & Maximum & Minimum & Average \\
\hline Blade & 176.46 & 2.69 & 56.82 \\
Hub and shroud & 165.94 & 0.76 & 67.82 \\
\hline
\end{tabular}

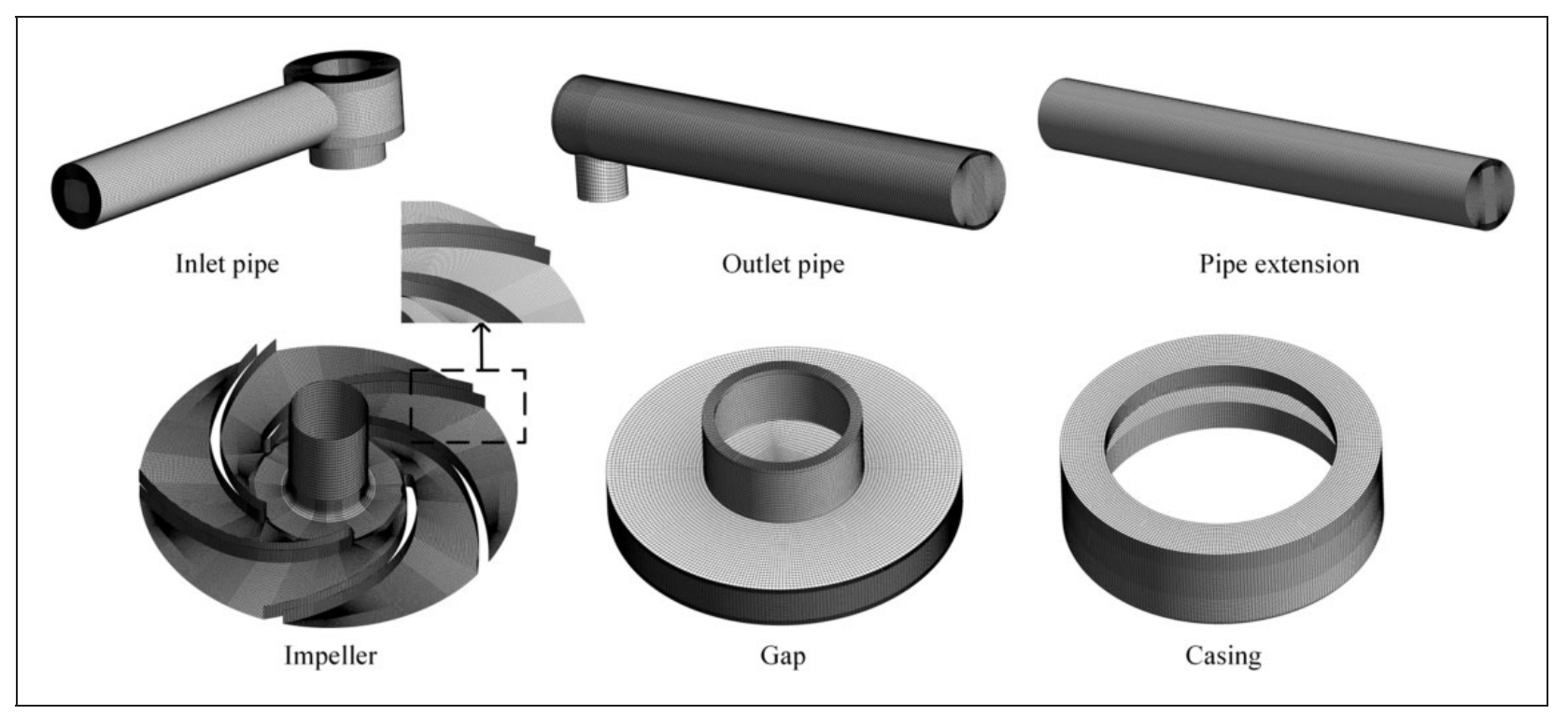

Figure 3. Mesh generation for different components. 
$3.3 \times 10^{-4} \mathrm{~s}$, corresponding to a runner-rotating angle of $2^{\circ}$ per time step.

\section{Results and discussions}

\section{Comparison between simulation and experiment}

The simulations in the current paper aim to analyze flow instability under several part load operation conditions. For comparison, the simulation with SST $k-\omega$ turbulence model has also been carried out. Further, the experimental measurements were conducted to obtain the pump performance and internal flow at Key Laboratory of Fluid Transmission Technology

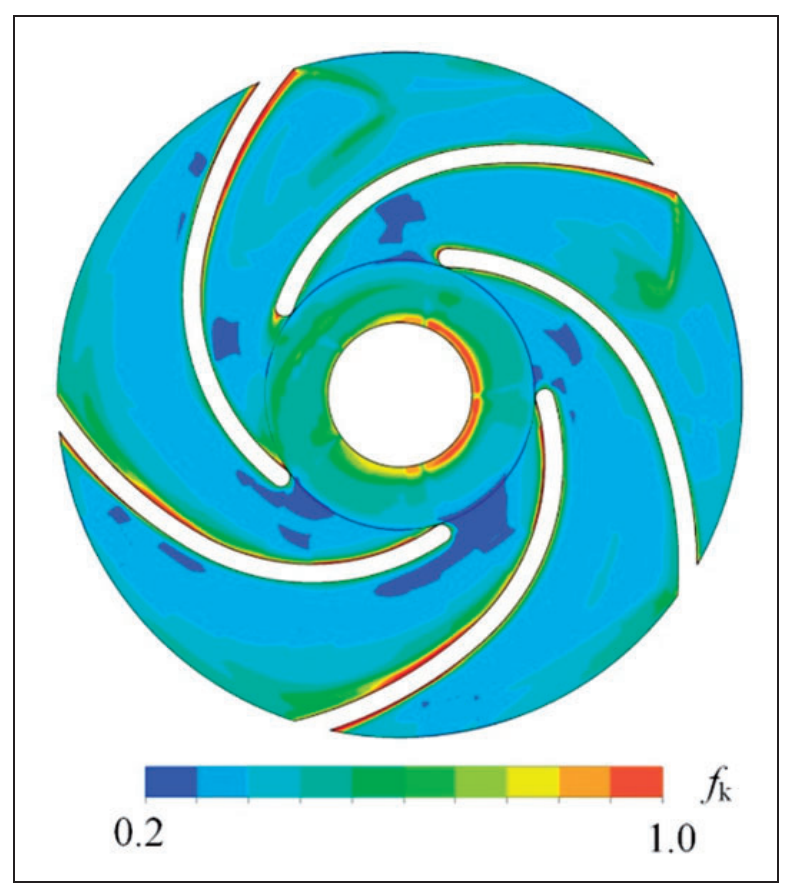

Figure 4. $f_{\mathrm{k}}$-Field by the mesh with 3.34 million grid points. of Zhejiang province, China. For the pressure sensors, the uncertainty is $\pm 0.5 \%$ at pump inlet and $\pm 0.1 \%$ at pump outlet. For the flow meter, the uncertainty is $\pm 0.1 \%$. The uncertainty of pump efficiency is less than $1 \%$.

For convenience, flow coefficient $\phi$ is defined in equation (9) to express the characteristic curve of the test pump

$$
\phi=\frac{Q}{\pi R_{2} b_{2} u_{2}}
$$

where $R_{2}$ stands for the impeller exit radius.

Figure 5 shows characteristic curves of the test pump. For better understanding, Figure 5(b) shows the characteristic curve from $\phi=0.008$ to $\phi=0.018$. It is clear that hydraulic performance is unstable when the pump is operated under $\phi=0.0087-0.018$. Note that for the numerical results, the averaged data are applied to show the head coefficient under each operation condition. The vertical bar under each operation condition presents head variation during the calculation. The comparison indicates that the predicted pump head is larger than the experimental value under each operation condition. For the averaged head prediction, the maximum relative error is $4.13 \%$ under the operation condition of $\phi=0.0051$ and the minimum relative error is $2.24 \%$ under design point of $\phi=0.0225$ with MPANS model. The comparison shows good agreement between the present numerical simulation and the experimental data.

Because the flow coefficient at best efficiency point i.e. $\phi_{\text {bep }}$ is 0.0225 , the range of unstable operation i.e. $\phi=0.0087-0.018$ is located under part load region for the test pump. Based on the experimental results of Figure 5, three unstable operation ranges can be clearly divided: the first at $0.39 \phi_{\text {bep }}$ $(\phi=0.0087)-0.55 \phi_{\text {bep }}(\phi=0.0124)$, the second at
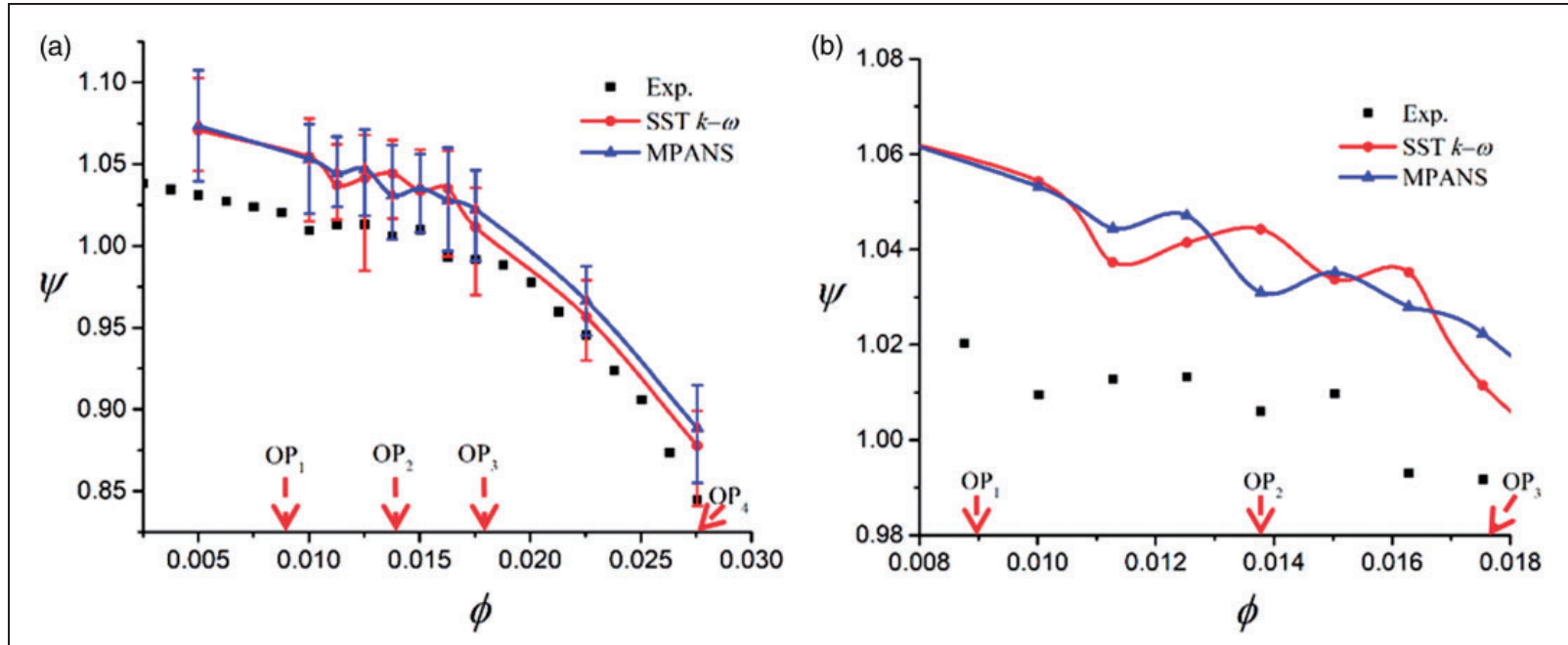

Figure 5. Characteristic curves of the test pump. (a) Overall discharge; (b) Part load discharge. 
$0.55 \phi_{\text {bep }}-0.66 \phi_{\text {bep }}(\phi=0.0150)$, and the third at $0.66 \phi_{\text {bep }}-0.78 \phi_{\text {bep }}(\phi=0.0175)$. As shown in Figure 5 (b), the prediction with MPANS model almost captured three breakdowns of pump head according to the experimental results. However, the prediction with SST $k-\omega$ turbulence model only captured two breakdowns of pump head. At two breakdown points of $\phi=0.0138$ and $\phi=0.0163$ according to the experimental data, the simulation with SST $k-\omega$ turbulence model estimated two local peaks respectively, and showed the different tendency of head drop. Thus, MPANS model predicts better tendency than SST $k$ $\omega$ model for the characteristic curves. In conclusion, it is applicable for MPANS to capture the unstable physics under part load conditions than SST $k-\omega$ turbulence model.

To make clear the differences between two turbulence models, the time-averaged contours of turbulence kinetic energy $(k)$ and eddy viscosity $\left(\mu_{\mathrm{t}}\right)$ at $\phi_{\text {bep }}$ on mid-span section of the impeller are shown for two turbulence models in Figure 6. It is noted that both turbulence kinetic energy and eddy viscosity of the main flow in blade-to-blade flow passage using SST $k$ - $\omega$ turbulence model are much larger than that using MPANS model. It is acknowledged that the conventional RANS approach usually overestimates the viscous effect of the turbulent flow, and is difficult to accurately predict the flow separation and other unstable flow phenomena. ${ }^{9}$ The overestimate of the viscous effect would be one important reason why SST $k-\omega$ model could not suitably capture the unstable flow in the test pump.

Figure 7 shows the time-averaged streamlines on mid-span section of the impeller by using MPANS model and SST $k-\omega$ turbulence model. For comparison, the available experimental images by PIV test are also supplied. Calibration has been conducted before the PIV test. In the figure, 3-part load conditions and 1 stable operation condition are chosen, and marked as $\mathrm{OP}_{1}-\mathrm{OP}_{4}$ as shown at Figure 5 , where $\mathrm{OP}_{1}$ represents the operation at $\phi=0.4 \phi_{\text {bep }}, \mathrm{OP}_{2}$ represents the operation under $\phi=0.6 \phi_{\text {bep }}, \mathrm{OP}_{3}$ represents the operation under $\phi=0.75 \phi_{\text {bep }}$, and $\mathrm{OP}_{4}$ represents the condition of $\phi=1.2 \phi_{\text {bep }}$.

As observed in the PIV experiments, vortices occur when the flow coefficient is lower than $0.75 \phi_{\text {bep }}$. The vortices are located at the impeller exit near both suction and pressure side of the blades. As the decrease of flow rate, the flow instability develops and the vortices expand in size. At $\mathrm{OP}_{1}\left(\phi=0.4 \phi_{\text {bep }}\right)$, each bladeto-blade passage is blocked off by two vortices, one is near the suction side of the blade while another is

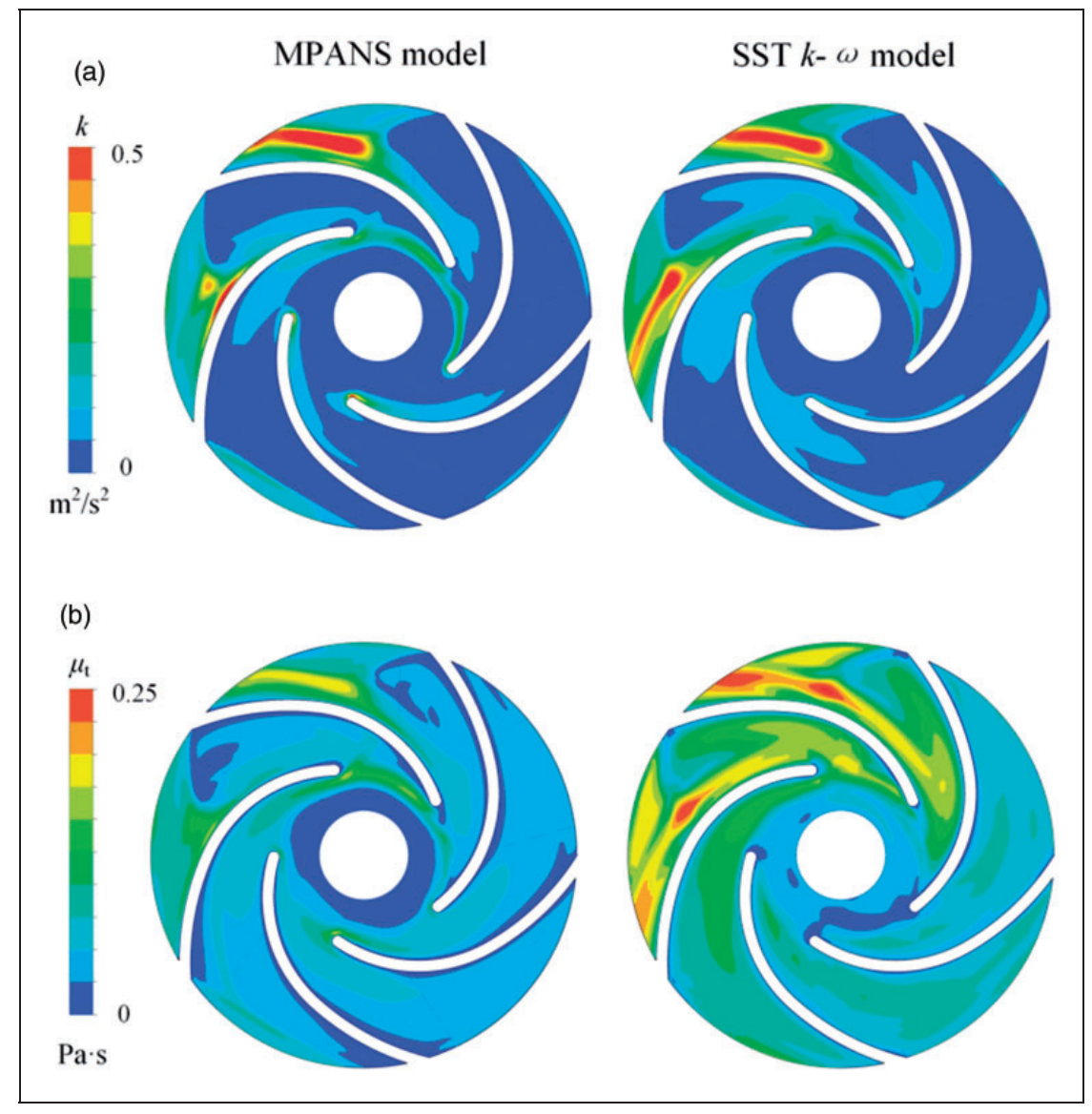

Figure 6. Turbulent kinetic energy and viscosity on mid-span section for two turbulence models. (a) Turbulence kinetic energy; (b) Eddy viscosity. 


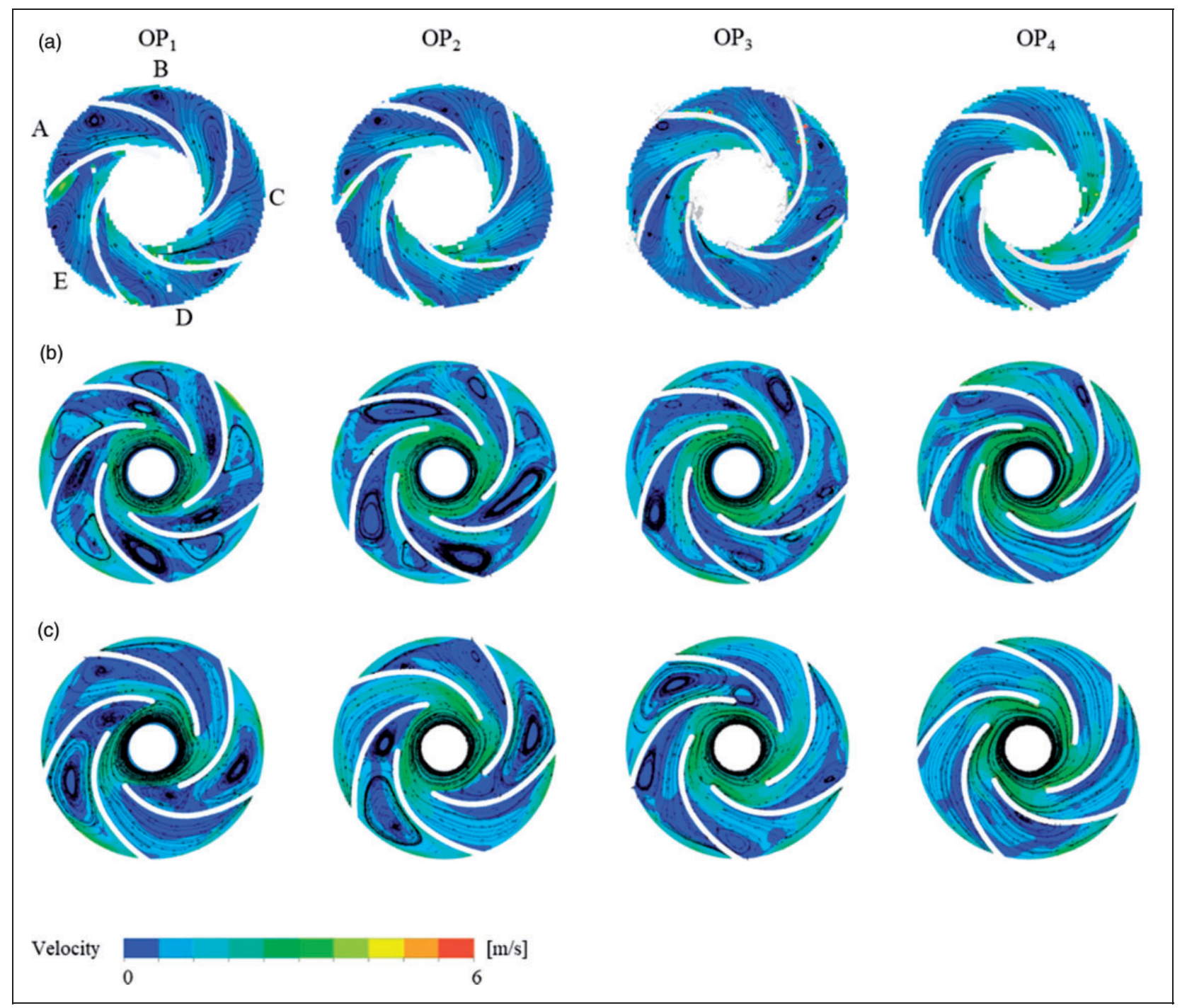

Figure 7. Time-averaged streamlines on mid-span section of the impeller among (a) experiments; (b) MPANS model; (c) SST $k$ - $\omega$ model.

near the pressure side of the blade. Note that MPANS model performs well and captures the similar flow features with the experiments results, while the vortices are only observed in blade-to-blade passage A, C, E by SST $k-\omega$ model. Additionally, there are some differences in the size and location of the vortices using SST $k-\omega$ model from the experimental data. Similar phenomena can be also observed under other part load conditions. At $\mathrm{OP}_{4}\left(\phi=1.2 \phi_{\text {bep }}\right)$, the streamlines are generally smooth as observed in the experiments. However, there is a small flow separation region near the blade pressure side of the blade-to-blade passage $\mathrm{D}$ predicted by SST $k-\omega$ model, while two small flow separation regions occur near the blade suction side in the blade-toblade passage A and B based on the simulation by MPANS model. Therefore, numerical simulation with both MPANS model and SST $k-\omega$ model can partly reproduce the complicated flow in the test pump. For the flow under the part-load conditions, MPANS model is preferable and helpful to depict the flow behaviors and reveal the mechanism of flow instability.

Thus, MPANS model is applied to investigate the internal flow in the test pump under part-load operation conditions in the following text.

\section{Time-averaged internal flow}

Figures 8 and 9 show the time-averaged internal flows by using MPANS model under four typical operation conditions.

Time-averaged vorticity in z-direction distributed on the mid-span section of impeller blade is displayed in Figure 8. It is noted that under the operation condition of $\phi=1.2 \phi_{\text {bep }}\left(\mathrm{OP}_{4}\right)$, the flow seems smooth within each blade-to-blade passage with no vortex blocked between two neighboring blades, though there are the zones with large value of vorticity near the leading edge of blade suction side due to slight flow separation. Further, the vorticity distribution is similar for each passage. For those part-load 


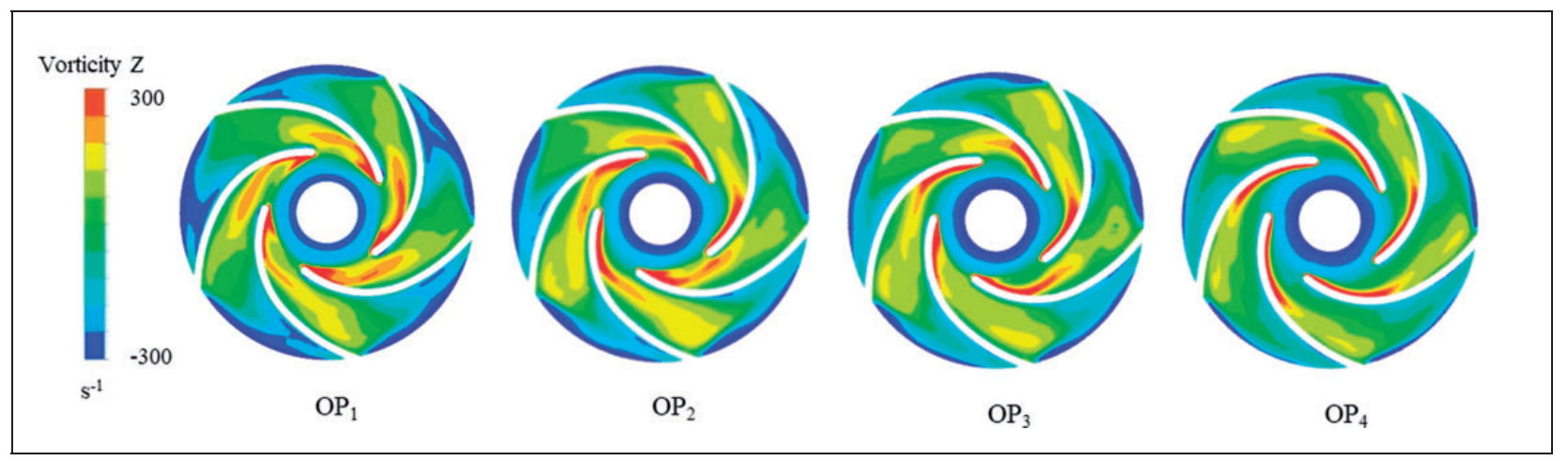

Figure 8. Time-averaged vorticity $Z$ contours on mid-span section of the impeller under four typical conditions.

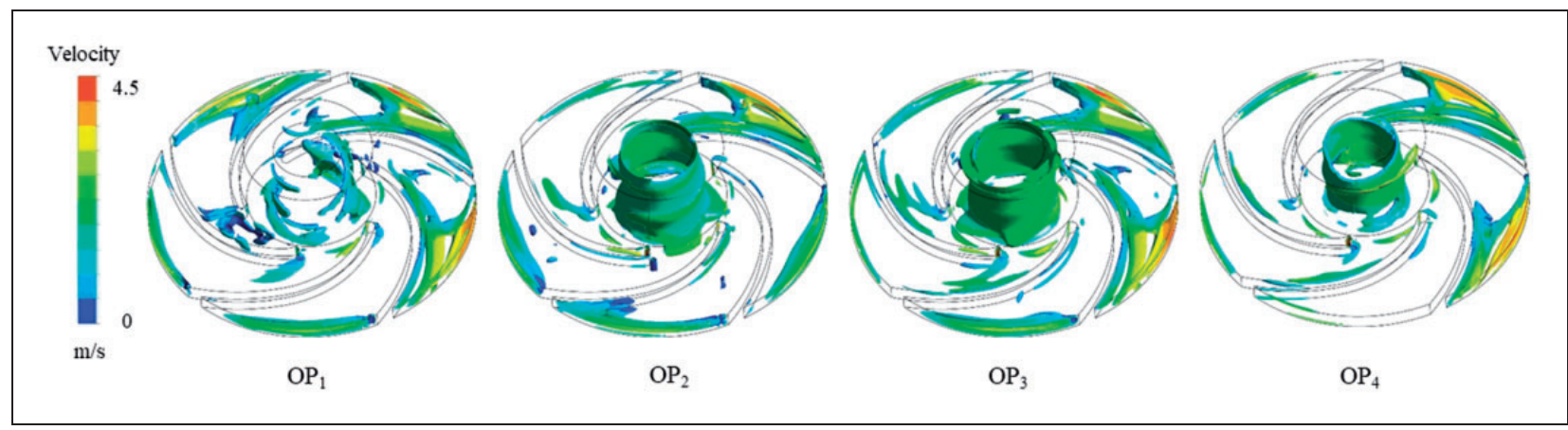

Figure 9. Iso-surface of $\lambda_{2}$-criterion on mid-span section of the impeller under four typical conditions.

conditions, the vortices attached to blade suction side at $\mathrm{OP}_{3}$ start to separate and develop to the main flow passage of the blade-to-blade channel. With the decrease of flow rate, the vortices with the reverse direction at the impeller exit become stronger, and extend to the flow passage inside the impeller. Under the condition of $\phi=0.4 \phi_{\text {bep }}\left(\mathrm{OP}_{1}\right)$, the highvorticity area almost blocks the inlet of all blade-toblade passages while the reverse vortices almost occupy the passages near impeller exit.

The vortex structures are illustrated by the iso-surface of $\lambda_{2}$-criterion colored with velocity magnitude as shown in Figure 9. As the velocity gradient tensor $(\nabla U)$ can be decomposed into symmetrical part $\left(S=\left(\nabla U \nabla U+\nabla U^{\mathrm{T}} \nabla U^{\mathrm{T}}\right) / 2\right)$ and asymmetric part $\left(\Omega=\left(\nabla U \nabla U-\nabla U^{\mathrm{T}} \nabla U^{\mathrm{T}}\right) / 2\right), \quad \lambda_{2}$ is defined as the second largest eigenvalue of $S^{2}+\Omega^{2}$. Note that if the value of $\lambda_{2}$ is below zero, the location in the flow field is a vortex core. ${ }^{24}$ In the current study, the flow separation induces rotating stall cell, which exhibits highly intensive vortex. In Figure 9, the value of $\lambda_{2}$ on the iso-surface is $-10200 \mathrm{~s}^{-1}$. Under the part-load condition, such as $\phi=0.4 \phi_{\text {bep }}\left(\mathrm{OP}_{1}\right)$, lots of vortex cores can be observed at the entrance of blade-toblade passage, pressure side of the blade and the impeller exit. In contrast, there is no strong vortex at the entrance of blade-to-blade passage under the operation condition of $\phi=1.2 \phi_{\text {bep }}\left(\mathrm{OP}_{4}\right)$, which is accordance with the former results, i.e., no clear

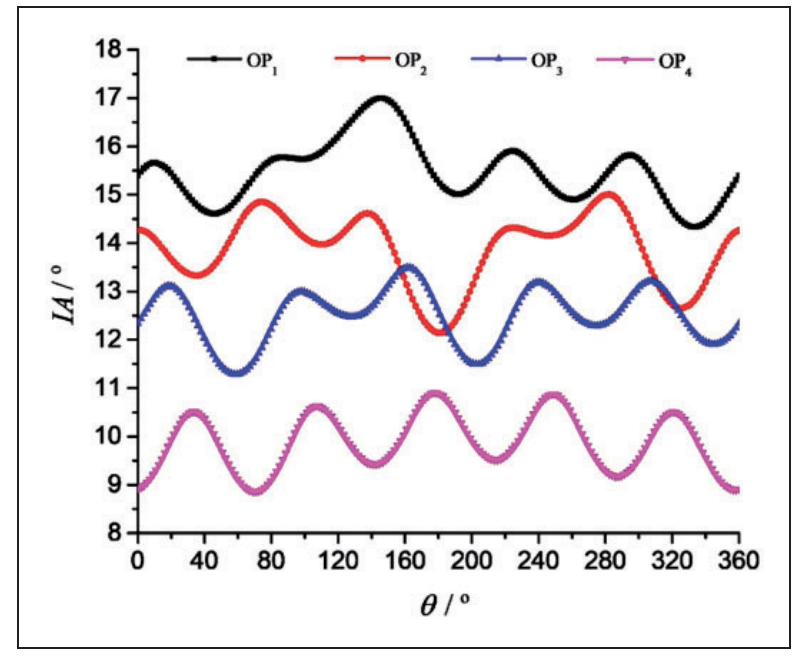

Figure 10. Incidence angle (IA) distributions under four typical conditions.

flow blockage at impeller inlet in Figure 7. Further discussion will be conducted at the section of Vorticity transport analysis.

The existence of large incidence angle (IA) plays a significance role in flow separation in a pump impeller as well as positive slope. ${ }^{25}$ Note that IA is calculated according to the blade angle and the relative flow direction at the blade leading edge. Figure 10 shows the distributions of time-averaged IA at the circumference 
of impeller inlet on mid-span section at different operation conditions. It is remarkable that the value of IA is near $9^{\circ}$ even at the condition of $\phi=1.2 \phi_{\text {bep }}\left(\mathrm{OP}_{4}\right)$. At the operation condition of $\phi=0.4 \phi_{\text {bep }}\left(\mathrm{OP}_{1}\right)$, the distribution of IA ranges from $14.5^{\circ}$ to $17^{\circ}$, which is large enough to cause flow separation at the leading edge of impeller blade. Further, for those part load conditions, IA has larger magnitude of vibrations.

In order to capture the characteristic of flow instability phenomenon, the variation of IA at a monitoring point in blade-to-blade passage $\mathrm{E}$ on the mid-span section are obtained as shown in Figure 11 in one shaft rotation period at the operation condition $\mathrm{OP}_{3}$. Note that the monitoring point for IA variation is marked by a red crosshair in Figure 12. The data at Figure 11 indicate that IA distributions range from $11.9^{\circ}$ to $17.1^{\circ}$ within one rotation period of pump shaft. The large variation may reflect the obvious vortex evolution accompanying with rotating stall, i.e. growth, mergence, development and shedding. For more details about the vortex evolution, three instants i.e., instant I, instant II and instant III are selected to reveal the relationship between the internal flow and vortex evolution in the impeller as shown in Figure 12.

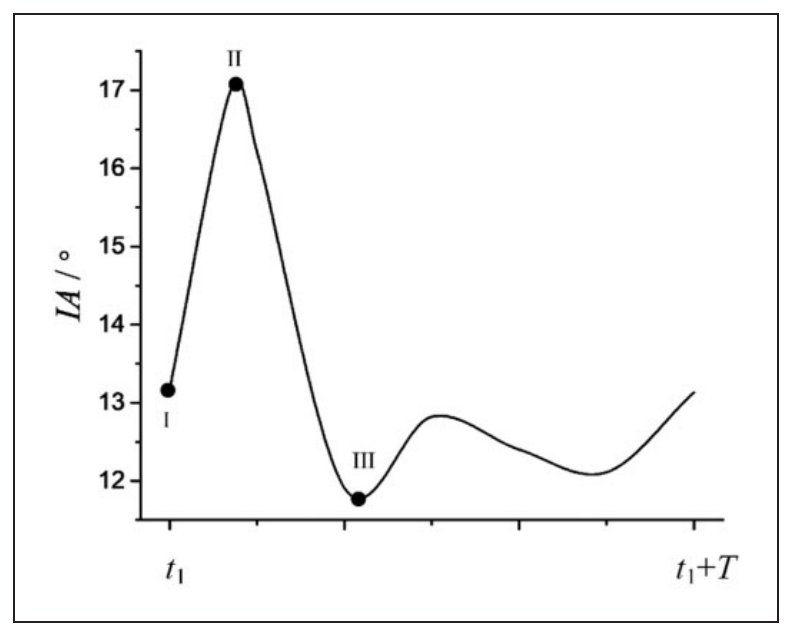

Figure II. IA distributions in one rotation period of pump shaft under the condition point $\mathrm{OP}_{3}$.
Figure 12 shows the streamline at three typical instants under the operation condition $\mathrm{OP}_{3}$. It is remarkable that the flow exhibits significant IA variation even at the same location owing to the evolution of rotating stall. At instant I where IA is $13.2^{\circ}$, the half of blade-to-blade passage $\mathrm{E}$ near suction side of a blade is occupied by the separation flow, and the flow seems fluent at the other part of the passage. At instant II, IA has the peak value of $17.1^{\circ}$. As demonstrated in the streamline distribution, the vortices merge and a larger separation zone forms in the blade-to-blade passage. At instant III, the value of IA reaches the minimum value, indicating the flow at the entrance of the passage is relative smooth among three instants. The result shown at Figure 12(c) can confirm this tendency. Therefore, it is evident that the obvious variation of IA will disturb the flow stability, which contributes to the flow separation in the flow passage, thus results in great energy loss and positive slope for pump performance. The great change of IA indicates the revolution of flow separation in blade-toblade passage is due to the movement of rotating stall.

\section{Vorticity transport analysis}

In order to achieve a deep insight into the mechanism of rotating stall, which is related with the movement of vortices in impeller passage, the vorticity transport equation is employed to analyze the internal flow as follows

$$
\begin{aligned}
\frac{D \vec{\omega}}{D t}= & (\vec{\omega} \cdot \nabla) \vec{V}-\vec{\omega}(\nabla \cdot \vec{V})+\frac{\nabla \rho_{m} \times \nabla p}{\rho_{m}^{2}} \\
& +\left(v_{m}+v_{t}\right) \nabla^{2} \vec{\omega}
\end{aligned}
$$

In equation (10), the first term on the right-handside (RHS) is the vortex stretching term by the velocity gradients, which represents the stretching and tilting of a vortex. The second term on the RHS is the vortex dilation owing to volumetric expansion/ contraction. Note that this term comes to zero when the fluid is incompressible. The third term on the RHS is the baroclinic torque due to the misaligned between

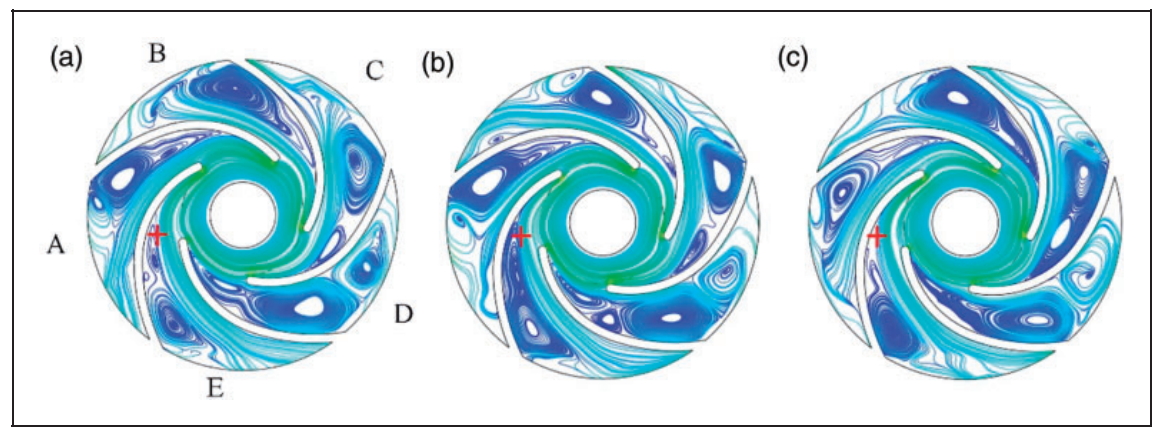

Figure 12. Streamlines on mid-span section under the condition $\mathrm{OP}_{3}$. (a) Instant I; (b) Instant II; (c) Instant III. 
density and pressure gradients. This term is negligible owing to no density change in the flow field. The last term on the RHS is the viscous dissipation due to viscous diffusion of the vorticity, which can be neglected owing to its smallest effect on the vortex transport equation in high Reynolds number flow. ${ }^{26}$ Thus, only the first term on the RHS is necessary for the analysis in the current study.

Figure 13 presents the predicted streamlines, isosurface of $\lambda_{2}$-criterion and contours of vortex stretching term on the mid-span section of the impeller within one rotating cycle under the operation condition $\mathrm{OP}_{3}$. The results show that there are strong vortex movement and great change rate of vortex in the pump impeller. As the overall feature, there are large vorticity intensity and vortex stretching term at the impeller exit and at blade leading edge. In the blade-to-blade flow passage $\mathrm{D}$, the streamlines are entangled and the passage is almost blocked off within the entire rotating circle. A large vortex is located at the center of the passage and attaches to both blades. Further, near the impeller exit there is a vortex cloud in the passage, corresponding with large vortex stretching term. It is also noted that this bladeto-blade passage is always blocked off, though there are vortex oscillations at the inlet and outlet of the passage. Within this rotating circle, the rotating stall is located in the blade-to-blade flow passage D. Since the blade-to-blade flow passage D is blocked off, the flow is pushed toward the next blade-to-blade passage. It is clear that the blade-to-blade flow passage E has larger velocity, especially near the blade pressure side according to Figure 13(a).

In the blade-to-blade passage $\mathrm{C}$, for $t=1 / 6 T$, a small flow separation occurs at the blade suction side, while a vortex near the inlet of the impeller moves downstream and begin to merge with the larger vortex. The same vortex cores are also observed by using iso-surface of $\lambda_{2}$-criterion. A low-speed vortex core is located near the impeller exit, which is due to the flow separation as shown by the streamline distribution. At the same location as the low-speed vortex core, the vortex stretching term has large magnitudes along the impeller exit as shown in Figure 13(c). For $t=2 / 6 T$ to $t=3 / 6 T$, the flow separation turns to be a small vortex splitting from the large one, and moves to the pressure side of the impeller. The vortex at inlet of the impeller merges with the large one in the blade-to-blade passage. Meanwhile, a small flow separation occurs at the pressure side of the blade with an increase magnitude in vortex stretching term shown in Figure $13(\mathrm{c})$. For $t=4 / 6 T$ to $t=5 / 6 T$, the split vortex moves to downstream and decays gradually as demonstrated in Figure 13(a). Near the pressure side of the outlet, the vortex stretching term decreases dramatically, with low magnitudes corresponding to the smooth streamlines. At the same time, a newly born flow separation seems to develop at the inlet of the impeller. At $t=6 / 6 T$, a small flow separation begins to develop at suction side of the blade and is going to split as displayed at $t=1 / 6 T$.

Based on those vortex behaviors, the propagation process of vortices in the impeller denotes strong flow separations occur in the blade-to-blade passage, and the vortices generate, merge with each other and split. The propagation of rotating stall makes the flow redistribution in blade-to-blade passages of the pump impeller. Furthermore, in the transient vortex flows, the distribution of vortex stretching term is of great accordance with the vortex cores, which has an important effect on vorticity transport.

\section{Pressure fluctuations}

The mechanism for the pressure fluctuations being excited by the rotating stall can be observed by means of setting pressure monitoring points in the impeller. The arrangement of three monitoring points is shown in Figure 14. Note that the monitoring point $\mathrm{P} 1$ is near the blade suction side and leading edge, and P3 is near the blade pressure side and trailing edge.

The non-dimensional parameter i.e. pressure coefficient is defined in equation (11).

$$
C \mathrm{p}=\frac{p_{\mathrm{i}}-\bar{p}}{0.5 \rho u_{2}^{2}}
$$

where $p_{\mathrm{i}}$ presents the instantaneous value of static pressure at a monitoring point, and $\bar{p}$ is the averaged pressure.

It is acknowledged that the pressure will oscillate dramatically as the stall cells move close a monitoring point. Figure 15 denotes pressure history at three monitoring points under the operation condition of $\phi=0.75 \phi_{\text {bep }}\left(\mathrm{OP}_{3}\right)$ where rotating stall cell can be observed. Note that parameter " $T$ " stands for one rotating cycle of the pump impeller.

Frequency spectrum analysis has been carried out to capture the rotating stall characteristics. Based on FFT (fast Fourier transform) method, the typical pressure oscillations with different frequencies are recognized as shown in Figure 16. At three monitoring points, two main peaks can be obtained with different frequency, i.e. $f=1.46 \mathrm{~Hz}$ and $f=16.67 \mathrm{~Hz}$. The component at $f=16.67 \mathrm{~Hz}$ is the rotational frequency of the impeller $\left(f_{\mathrm{n}}\right)$. The dominant component is regarded as the pressure oscillation due to rotating stall, whose frequency $\left(f_{\text {stall }}\right)$ is $f=1.46 \mathrm{~Hz}$, much less than the rotational frequency. The result confirmed that the evolution cycle of rotating stall was approximately 12 times the period of shaft rotation i.e., $T$, as depicted in our previous study. ${ }^{27}$ At the monitoring points $\mathrm{P} 1$ and $\mathrm{P} 2$, the $f_{\text {stall }}$ component has much larger amplitude than the $f_{\mathrm{n}}$ component, and at the monitoring points $\mathrm{P} 3$ there is identical amplitude for $f_{\text {stall }}$ and $f_{\mathrm{n}}$ component. It is also noted that $f_{\text {stall }}$ is much lower than $f_{\mathrm{n}}$, i.e., $f_{\text {stall }}=8.76 \% f_{\mathrm{n}}$. That means rotating stall cells propagate in the impeller with a much slower 


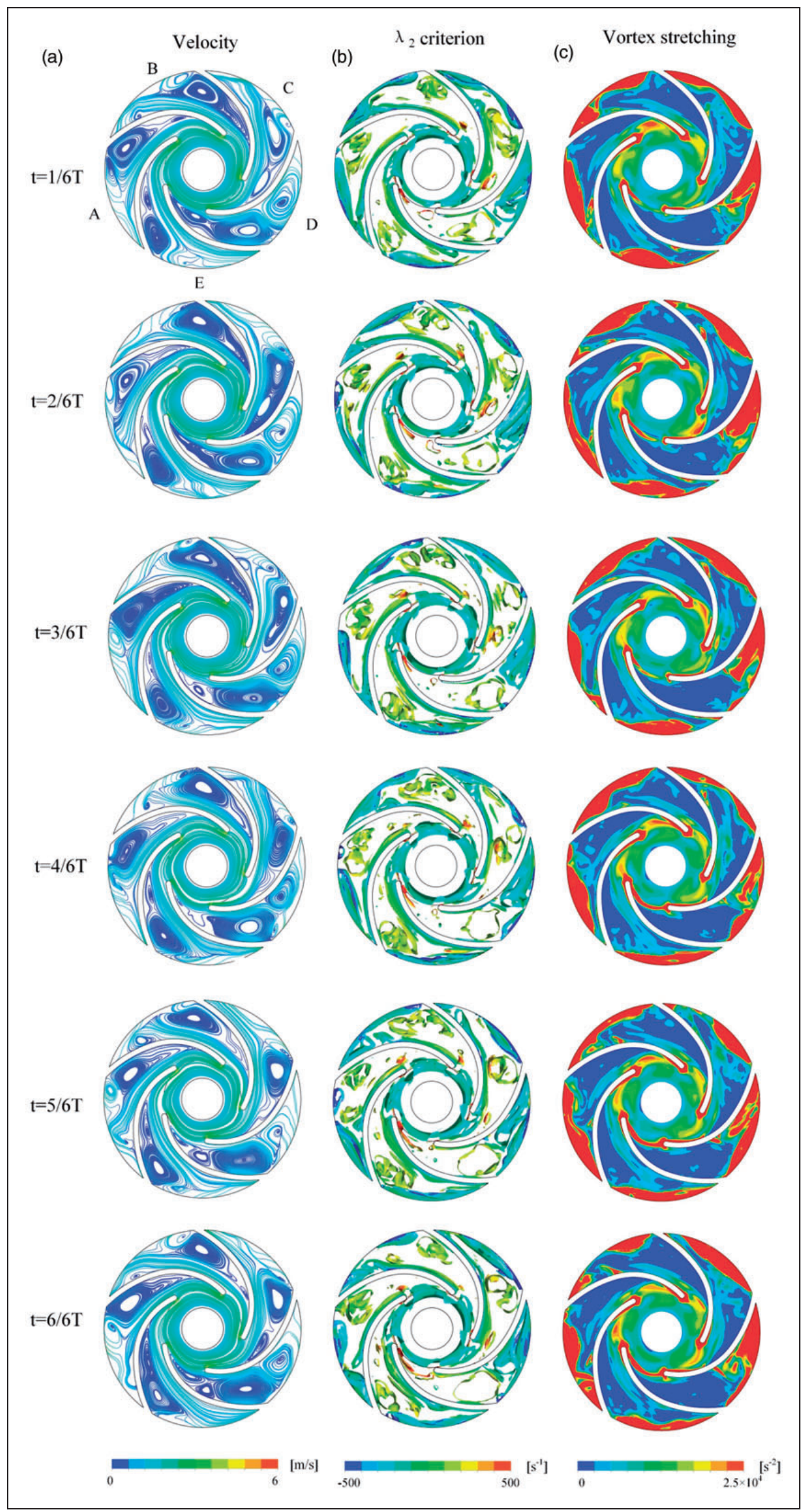

Figure 13. Flow on mid-span section of the impeller under the condition point $\mathrm{OP}_{3}$ : (a) streamlines, (b) iso-surface of $\lambda_{2}$-criterion, (c) vortex stretching term. 


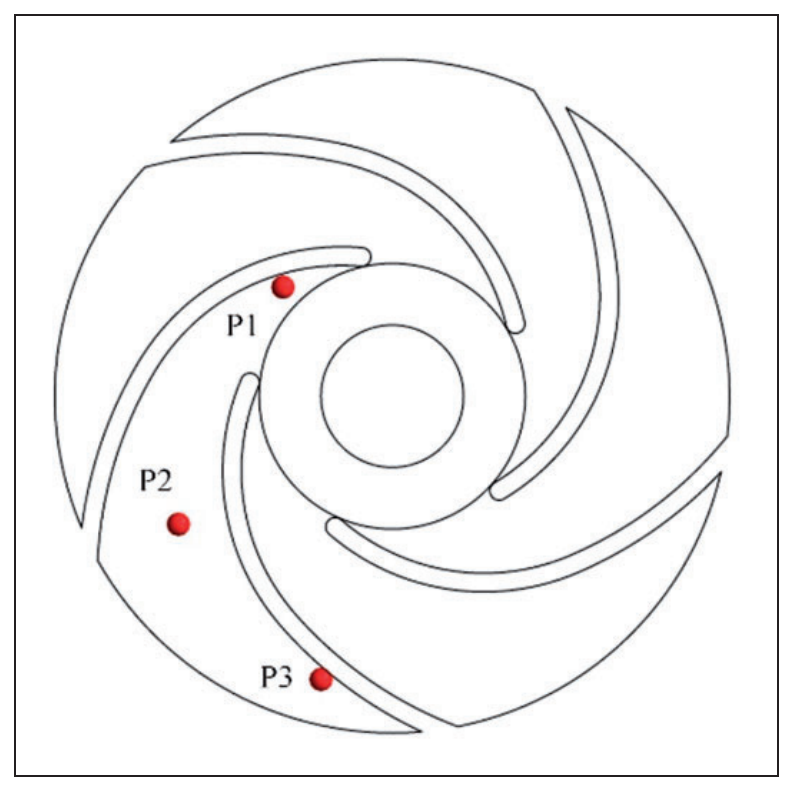

Figure 14. The location of the monitoring points.

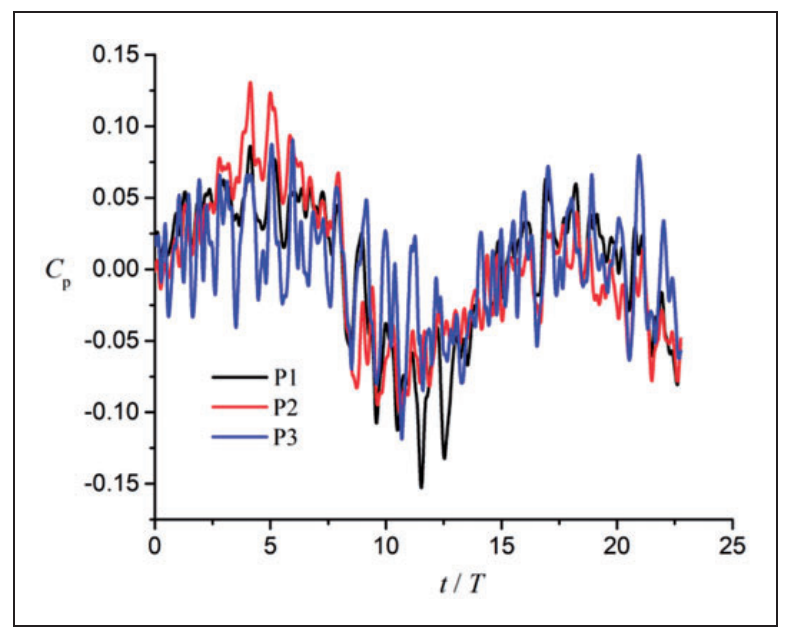

Figure I5. Pressure history distribution at three monitoring points.

speed. Thus, the period of the rotating stall is 11.4 times of impeller rotating circle. As shown at Figure 13, the rotating stall cell always stays within the blade-to-blade passage $\mathrm{D}$ in one impeller rotating circle.

\section{Lagrangian coherent structure}

It is clear that Lagrangian coherent structure (LCS) is helpful to depict the separation flow. ${ }^{22}$ In the study of LCS, Finite-Time Lyapunov Exponent (FTLE) refers to the averaged separation rate of particle trajectories during a finite time interval, and LCS is defined as the ridge of the Finite-Time Lyapunov Exponent (FTLE). In two-dimensional flow, for a fluid particle with

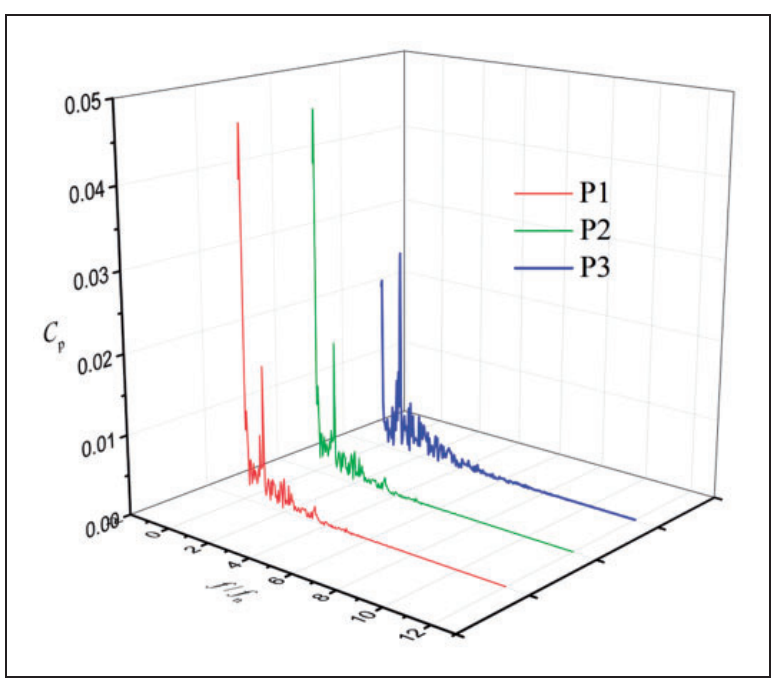

Figure 16. Pressure fluctuations in frequency domain at three monitoring points.

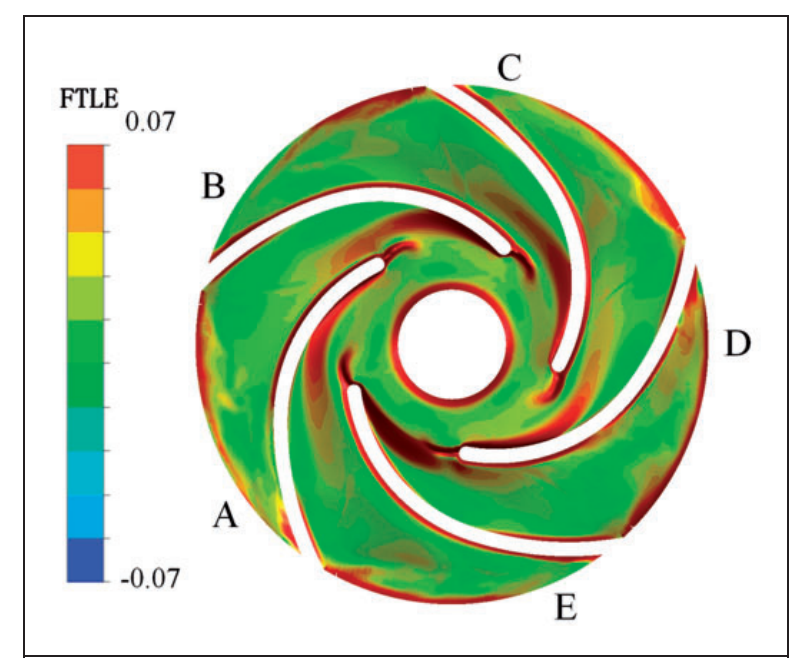

Figure 17. FTLE distribution on mid-span section.

initial position $\left(\mathrm{x}_{0}, \mathrm{y}_{0}\right)$ at time instant $t=t_{0}$ and final position $\left(\mathrm{x}_{\mathrm{T}}, \mathrm{y}_{\mathrm{T}}\right)$ at time instant $t=t_{0}+T$, Haller and Yuan ${ }^{17}$ defined the FTLE as

$$
\Delta x=\frac{1}{|T|} \ln \left(\sqrt{\lambda_{\max }\left(\varphi^{T} \times \varphi\right)}\right)
$$

where $\lambda_{\max }\left(\varphi^{T} \times \varphi\right)$ is the maximum eigenvalue of Cauchy-Green deformation tensor, which represents the maximum extension of the particle. And $\varphi$ is defined as the deformation gradient tensor which can be written as

$$
\varphi=\left[\begin{array}{ll}
\frac{d x_{T}}{d x_{0}} & \frac{d x_{T}}{d y_{0}} \\
\frac{d y_{T}}{d x_{0}} & \frac{d y_{T}}{d y_{0}}
\end{array}\right]
$$

In addition, $\varphi^{T}$ is the transpose of $\varphi$. 
Figure 17 presents FTLE distributions in one stall cycle, which indicates the boundary of the flow separation on mid-span section of the impeller under the operation condition of $\mathrm{OP}_{3}$. As shown in Figure 17, the value of FTLE near blade leading edge and the impeller exit is relatively high, which indicates strong separation flows are likely to occur at the entrance and the impeller exit for each blade-to-blade passage. The entrance of blade-to-blade passage $\mathrm{D}$ is closed by the cluster of FTLE with large magnitude. Those

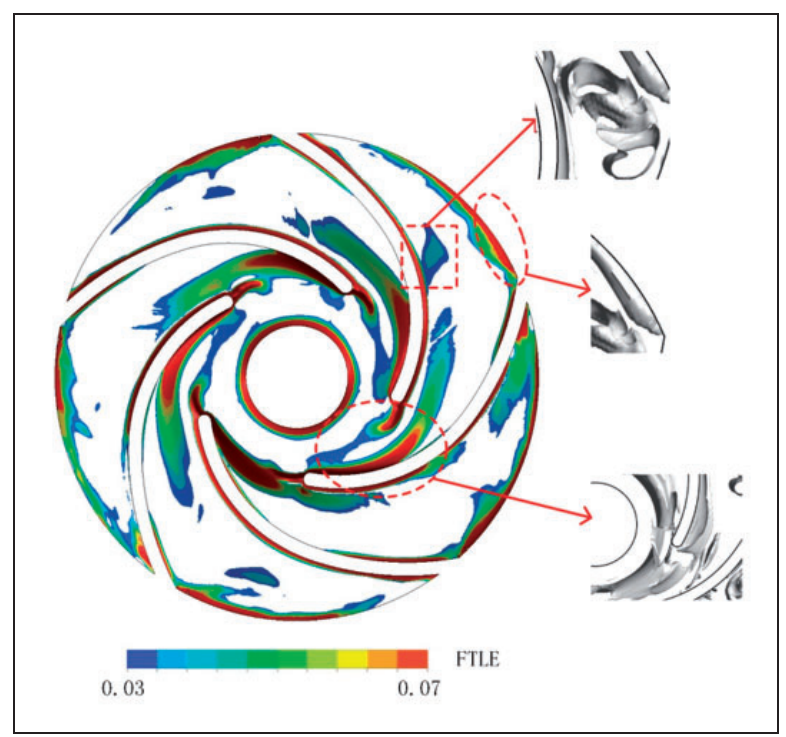

Figure 18. LCS on mid-span section. results have good agreement with that shown at Figure 13(c).

Details about LCS on the mid-span section are shown in Figure 18, where iso-surface of $\lambda_{2}$-criterion is also included for blade-to-blade passage $\mathrm{C}$. The value of FTLE near blade leading edge and impeller exit is higher than that in blade-to-blade passage. It is noted that rotating stall induces large-scale separation in entire region of the pump impeller. In blade-toblade passage $\mathrm{C}$, LCS is located near the passage entrance, the middle of blade pressure side and the impeller exit. For LCS originated from the blade suction side, it develops toward the center of the main stream, and almost attaches to the blade pressure side. Among five blade-to-blade passages, the flow separation is the most severe in blade-to-blade passage D, where the entrance and the exit of the passage are closed by LCS.

To present the flow separation phenomenon and transient process of the vortex dynamic, tracer particles are initially set at leading edge of the impeller blade and the impeller exit as shown in Figure 19. Different colors represent the tracer at different time instant. Basically, the flow in the passage is not fluent. Near the blade leading edge, five tracer particles are set as shown in Figure 19(a). "track 1" seems stagnant and no clear displacement with $12 T$; "track 2 " and "track 3" move slowly and reach near the blade finally; "track 4" and "track 5" flow along the blade suction side, and still in the passage even after the long period of $12 T$. As illustrated in Figure 19(b), tracer particles set at the impeller exit move with very slow velocity, and almost flock near the blade pressure side. The tracer distribution shows the history of the

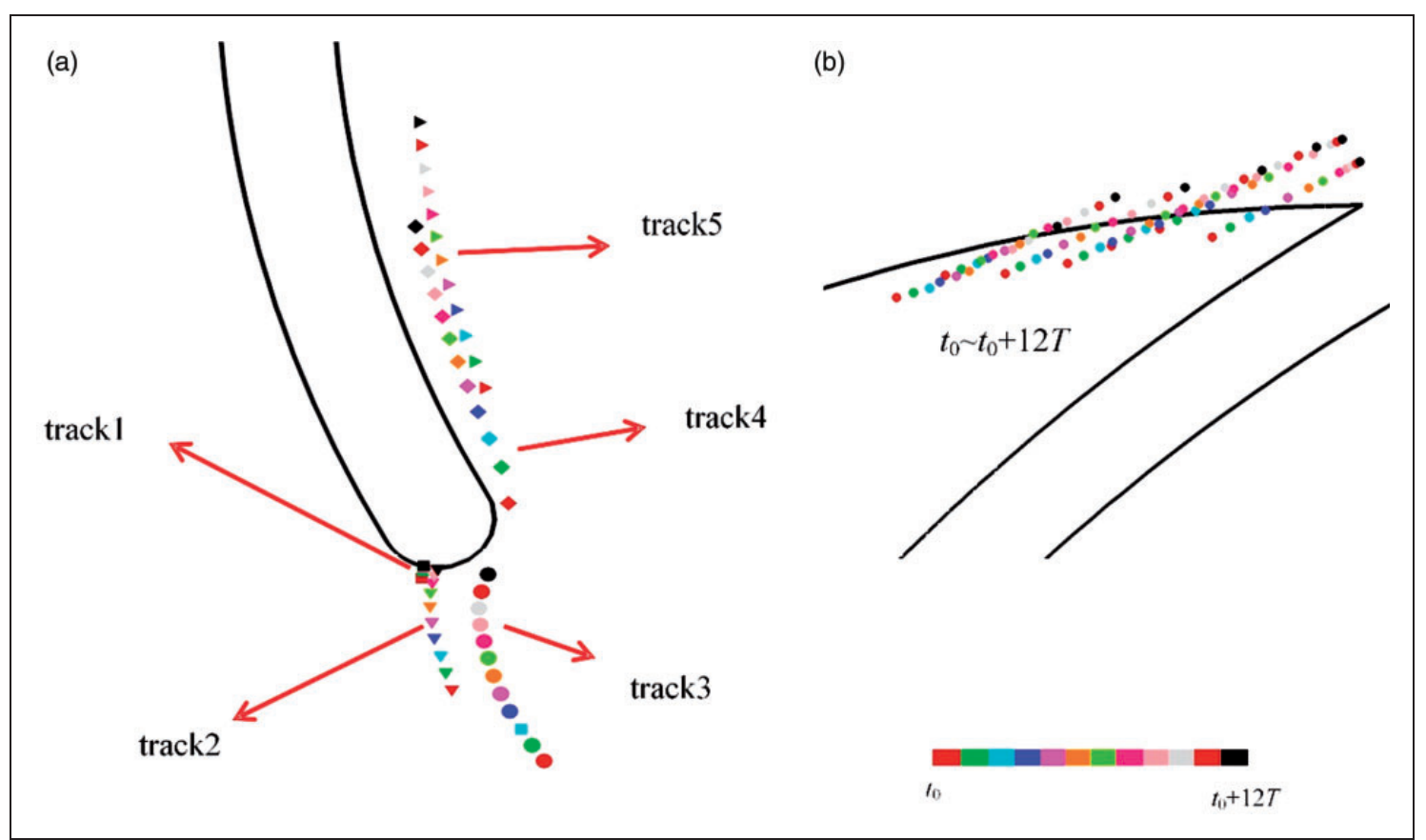

Figure 19. The movement of tracer particles at different locations. (a) Near blade leading edge; (b) Near impeller exit. 
fluid particles, indicating the flow blockage due to the strong separation as well as the vortex movement in the present passage.

\section{Concluding remarks}

In this paper, modified partially averaged NavierStokes (MPANS) turbulence model based on the standard $k-\varepsilon$ model is performed to predict the flow instability in a low specific speed centrifugal pump. The time-averaged internal flow, flow incidence at the entrance of blade-to-blade passage, vortex evolution and pressure fluctuations due to rotating stall, etc. are discussed for different operation conditions. Furthermore, based on Lagrangian viewpoint, two-dimensional FTLE and LCS are used to analyze the separation flow in blade-to-blade passages of the pump impeller. Conclusions can be drawn as follows:

(1) Both characteristic curves and time-averaged internal flow predicted by the modified PANS model are in good agreement with the available experimental data. When compared with the SST $k$ - $\omega$ model, MPANS model shows better performance for investigating the flow in the current pump under part-load conditions.

(2) The flow exhibits significant incidence angle variation at the entrance of blade-to-blade passage in the pump impeller owing to the strong separation flow as well as vortex evolution. In addition, the evolution period of the rotating stall is around 11.4 times of the pump rotation period.

(3) During the evolution of rotating stall, the separation flow occurs at blade suction side and develops from the leading edge to the main passage in the impeller. The mechanism of strong separation in the flow passage is originated from the transition of rotating stall, which is associated with vorticity transport. It is also noted that the vortex stretching term has an important effect on vorticity transport under the part-load operation conditions.

(4) Two-dimensional FTLE and LCS are helpful to show the flow separation in blade-to-blade passage. Both LCS and vortex structure by $\lambda_{2}$ can clearly depict the passage blockage and flow separation due to rotating stall under part-load operation conditions.

(5) Pressure fluctuations are excited by the periodic evolution of rotating stall. According to the frequency spectrum analysis, the rotating stall frequency $\left(f_{\text {stall }}\right)$ is much lower than the pump rotation frequency $\left(f_{\mathrm{n}}\right)$, i.e. $f_{\text {stall }}=8.76 \% f_{\mathrm{n}}$.

\section{Declaration of Conflicting Interests}

The author(s) declared no potential conflicts of interest with respect to the research, authorship, and/or publication of this article.

\section{Funding}

The author(s) disclosed receipt of the following financial support for the research, authorship, and/or publication of this article: This work was financially supported by National Key R\&D Program of China (2018YFB0606101), the National Natural Science Foundation of China (grant no. 51536008), Beijing Natural Science Foundation (3182014), Science and Technology on Water Jet Propulsion Laboratory (project no. 61422230103162223004), State Key Laboratory for Hydroscience and Engineering (project no. sklhse-2017-E02) and Tsinghua National Laboratory for Information Science and Technology.

\section{ORCID iD}

Weixiang Ye (D) http://orcid.org/0000-0002-6740-3192

Xiaojun Li (D) http://orcid.org/0000-0002-5530-1666

\section{References}

1. Luo XW, Ji B and Tsujimoto Y. A review of cavitation in hydraulic machinery. $J$ Hydrodyn 2016; 28: 335-358.

2. Gu GX, Banda S and Sparks A. An overview of rotating stall and surge control for axial flow compressors. IEEE Trans Control Syst Technol 1999; 7: 639-647.

3. Brennen CE. Hydrodynamics of pumps. Oxford: Concepts ETI, Inc. and Oxford University Press, 1994, pp.169-208.

4. Ran HJ, Luo XW, Zhu L, et al. Experimental study of the pressure fluctuations in a pump turbine at large partial flow conditions. Chin J Mech Eng 2012; 25: 1205-1209.

5. Ullum U, Wright J, Dayi O, et al. Prediction of rotating stall within an impeller of a centrifugal pump based on spectral analysis of pressure and velocity data. $J$ Phys Conf Ser 2006; 52: 36-45.

6. Krause N, Zähringer K and Pap E. Time-resolved particle imaging velocimetry for the investigation of rotating stall in a radial pump. Exper Fluids 2005; 39: 192-201.

7. Walters DK and Cokljat D. A three-equation eddyviscosity model for Reynolds-averaged Navier-Stokes simulations of transitional flow. J Fluids Eng 2008; 130: $320-327$.

8. Li XJ, Zhu ZC, Li Y, et al. Experimental and numerical investigations of head-flow curve instability of a singlestage centrifugal pump with volute casing. Proc IMechE, Part A: J Power and Energy 2016; 230: 633-647.

9. Huang RF, Luo XW and Ji B. Numerical simulation of the transient cavitating turbulent flows around the Clark-Y hydrofoil using modified partially averaged Navier-Stokes method. J Mech Sci Technol 2017; 31: 2849-2859.

10. Zhou PJ, Wang FJ, Yang ZJ, et al. Investigation of rotating stall for a centrifugal pump impeller using various SGS models. J Hydrodyn 2017; 29: 235-242.

11. Li DY, Han L, Wang HJ, et al. Pressure fluctuation prediction in pump mode using large eddy simulation and unsteady Reynolds-averaged Navier-Stokes in a pump-turbine. Adv Mech Eng 2016; 8: 1-12.

12. Girimaji SS. Partially-averaged Navier-Stokes model for turbulence: a Reynolds-averaged Navier-Stokes to 
direct numerical simulation bridging method. $J$ Appl Mech 2006; 73: 413-421.

13. Lakshmipathy S and Girimaji SS. Partially averaged Navier-Stokes (Pans) method for turbulence simulations: flow past a circular cylinder. J Fluids Eng 2010; 132: 121202 .

14. Luo XW, Huang RF and Ji B. Transient cavitating vertical flows around a hydrofoil using $k-\omega$ partially averaged Navier-Stokes model. Modern Phys Lett B 2016; 30: 1550262.

15. Abdol-Hamid KS and Girimaji SS. A Two-stage procedure toward the efficient implementation of PANS and other hybrid turbulence models. NASA Technical Report no. TM 2004, 213260.

16. Huang RF, Luo XW, Ji B, et al. Turbulent flows over a backward facing step simulated using a modified partially averaged Navier-Stokes model. J Fluids Eng 2017; 139: 044501 .

17. Haller $\mathrm{G}$ and Yuan G. Lagrangian coherent structures and mixing in two-dimensional turbulence. Physica $D$ 2000; 147: 352-370.

18. Franco E, Pekarek DN, Peng JF, et al. Geometry of unsteady fluid transport during fluid-structure interactions. J Fluid Mech 2007; 589: 125-145.

19. Green MA, Rowley CW and Haller G. Detection of Lagrangian coherent structures in three-dimensional turbulence. J Fluid Mech 2007; 572: 111-120.

20. Kasten J, Petz C, Hotz I, et al. Lagrangian feature extraction of the cylinder wake. Phys Fluids 2010; 22: 3365-3274.

21. Tseng $\mathrm{CC}$ and $\mathrm{Hu} \mathrm{HA}$. Flow dynamics of a pitching foil by Eulerian and Lagrangian viewpoints. AIAA J 2015; 54: 1-16.

22. Yang DD, Yu A, Ji B, et al. Numerical analyses of ventilated cavitation over a 2D NACA0015 hydrofoil using two turbulence modeling methods. $J$ Hydrodyn 2018; 30: 345-356.

23. Long XP, Cheng HY, Ji B, et al. Large eddy simulation and Euler-Lagrangian coupling investigation of the transient cavitating turbulent flow around a twisted hydrofoil. Int $J$ Multiphas Flow 2018; 100: 41-56.

24. Jeong $J$ and Hussain $F$. On the identification of a vortex. J Fluid Mech 1995; 285: 69-94.

25. Yang DD, Luo XW, Liu DM, et al. Unstable flow characteristics in a pump-turbine simulated by a Modified Partially-Averaged Navier-Stokes method. Sci Chin Technol Sci 2018; 61.

26. Ji B, Luo XW, Arndt REA, et al. Numerical simulation of three dimensional cavitation shedding dynamics with special emphasis on cavitation-vortex interaction. Ocean Eng 2014; 87: 64-77.

27. Ye WX, Huang RF, Jiang ZW, et al. Instability analysis under part-load conditions in centrifugal pump. J Mech Sci Technol 2019; 33: 269-278.

\section{Appendix}

\section{Notation}

\begin{tabular}{|c|c|}
\hline bep & best efficiency point \\
\hline$C_{\mathrm{p}}$ & pressure coefficient \\
\hline$C_{\varepsilon 1}, C_{\varepsilon 2}$ & turbulence model constants \\
\hline$C_{\mu}$ & model coefficient \\
\hline$f_{\mathrm{k}}$ & $\begin{array}{l}\text { unresolved-to-total ratio of kinetic } \\
\text { energy }\end{array}$ \\
\hline$f_{\mathrm{n}}$ & frequency of the impeller \\
\hline$f_{\text {stall }}$ & frequency of rotating stall \\
\hline$f_{\varepsilon}$ & unresolved-to-total ratio of dissipation \\
\hline$H$ & pump head \\
\hline$k, k_{\mathrm{u}}$ & $\begin{array}{l}\text { total and unresolved turbulence kinetic } \\
\text { energy }\end{array}$ \\
\hline$M$ & the torque of the rotating shaft \\
\hline$p$ & mixture pressure \\
\hline$p_{\mathrm{i}}, \bar{p}$ & $\begin{array}{l}\text { instantaneous and averaged value of } \\
\text { static pressure }\end{array}$ \\
\hline$Q$ & flow rate \\
\hline$R_{2}$ & radius at impeller exit \\
\hline$T$ & one rotating cycle of the pump impeller. \\
\hline$u_{\mathrm{i}}$ & velocity in the $i$-th direction \\
\hline$u_{\mathrm{j}}$ & velocity in the $j$-th direction \\
\hline$u_{2}$ & peripheral velocity at impeller exit \\
\hline$\Delta$ & the local grid size \\
\hline$\varepsilon_{\mathrm{u}}$ & unresolved turbulence dissipation \\
\hline$\Lambda$ & Taylor turbulence length scale \\
\hline$\mu$ & laminar dynamic viscosity \\
\hline$\mu_{\mathrm{t}}$ & turbulence dynamic viscosity \\
\hline$v$ & laminar kinematic viscosity \\
\hline$v_{\mathrm{t}}$ & turbulence kinematic viscosity \\
\hline$\rho_{\mathrm{m}}$ & mixture density \\
\hline & angular speed \\
\hline$\nabla U, \nabla U^{\mathrm{T}}$ & $\begin{array}{l}\text { velocity gradient tensor and its } \\
\text { transpose }\end{array}$ \\
\hline
\end{tabular}

